\title{
Revaluing the role of the tongue in obstructive sleep apnea
}

\author{
Michel Burihan Cahali1,2,a
}

Since the earliest descriptions of obstructive sleep apnea (OSA), researchers have been struggling to determine the location and pattern of airway collapse in this disease. From the early general notion of upper airway apnea(1) to the most recent detailed classifications of the patterns of collapse seen on drug-induced sleep endoscopy (DISE), (2) understanding the complex mechanical behavior of the upper airway during sleep in individuals with OSA remains a challenge and provides an opportunity to advance the medical and surgical treatment of OSA.

In this issue of the JBP, Passos et al. ${ }^{(3)}$ present the results of multislice computed tomography of the airway in patients with OSA and healthy controls (mean apnea-hypopnea index of 57.1 events/h and 2.2 events/h, respectively) during wakefulness and monitored natural sleep. In the latter case, all control subjects maintained stable breathing during image acquisition, whereas the sleepstate images of the OSA group subjects were acquired during episodes of obstructive apnea or hypopnea, as confirmed by polysomnography. From wakefulness to sleep, there was no significant reduction in the space behind the tongue (retroglossal space) in either group, although there was a significant decrease in the space behind the soft palate (retropalatal space) in the OSA group. The retropalatal space showed a significant reduction in its anteroposterior and lateral dimensions.

To understand the dynamic changes in the airway that lead to obstructive events, Passos et al.(3) evaluated the structures surrounding the pharynx. During sleep, the volume of the lateral pharyngeal wall increased significantly in OSA group subjects and did not change in control group subjects. In sagittal reconstructions, the authors measured the distance from the posterior third of the part of the tongue that lies in front of the soft palate (i.e., the oral tongue) to the posterior pharyngeal wall, which they designated the tongue-pharyngeal distance. Because that distance decreased significantly from wakefulness to sleep in the OSA group subjects, the authors suggested that the retropalatal narrowing is caused by enlargement of the lateral pharyngeal wall associated with the posterior displacement of the upper part of the tongue.

Studies involving imaging of the upper airway during sleep with concurrent polysomnography are still quite rare in the literature. ${ }^{(4)}$ Therefore, Passos et al. ${ }^{(3)}$ are to be commended. The images in their study were obtained during natural sleep rather than by DISE. In addition, the authors were able to quantify the dynamic changes in the pharyngeal lumen and surrounding tissues by computed tomography. This appears to be a nearly perfect setting to study the mechanical behavior of the factors responsible for obstructing the upper airway in OSA. Given the information provided by the authors, the roles played by those factors need to be revalued.

The pharyngeal structures are integrated, consisting of multiple layers of muscle fibers with different origins, insertions, and fusions. ${ }^{(5,6)}$ Ultimately, the change in the shape of the pharynx from the waking state to the sleep state depends on complex interactions among the tissues of the lateral pharyngeal wall, soft palate, and tongue, as well as the non-negligible effect of the jaw opening in the whole scenario. ${ }^{(7)}$ Hence, the levels of obstruction described in DISE examinations do not necessarily represent independent sources of obstruction. For instance, the palatopharyngeus muscle connects the soft palate to the lateral pharyngeal wall and forms the posterior tonsillar pillar, whereas the palatoglossus muscle connects the soft palate to the lateral wall-forming the anterior tonsillar pillar/palatoglossal arch-and extends to the lateral base of the tongue. In fact, the lateral pharyngeal wall is the site of insertion of the soft palate into the pharynx. Not surprisingly, Passos et al. (3) found that, from wakefulness to sleep, lateral pharyngeal wall enlargement correlated significantly with retropositioning of the soft palate in all of the subjects studied.

Passos et al. ${ }^{(3)}$ speculated that, during sleep, the posterior displacement of the tongue would relax the palatoglossal arch and allow the folding and consequent increase in the volume of the lateral wall, as well as pushing the soft palate backwards, all of which, together, cause circumferential narrowing of the retropalatal airway. In that hypothesis, the tongue represents the leading mechanism of obstruction and causes the changes in the other pharyngeal structures. The data presented in the current Passos et al. ${ }^{(3)}$ study are so detailed that we can certainly consider a completely different mechanism. There was absolutely no significant narrowing of the retroglossal space during the obstructive events. In the patients with very severe OSA, among whom the mean body mass index was $34.5 \mathrm{~kg} / \mathrm{m}^{2}$, the base of the tongue moved backward a mean of only $1 \mathrm{~mm}$ from wakefulness to obstructed sleep! Because the palatoglossal arch is located precisely in that space, below the level of the soft palate, it seems unlikely that the tongue causes the lateral wall folding, either through relaxation of its interconnections or by directly pushing the lateral wall. The question of whether the oral tongue causes retropalatal collapse is debatable. Among all of the subjects studied, the backward movement of the soft palate from the 
waking state to the sleep state correlated well with that of the oral tongue ( $r=0.77)$, and the thickness of the soft palate did not change significantly in the OSA group, suggesting a synchronized movement of those tissues rather than a push between them. In contrast, the volume of the parapharyngeal fat pad decreased significantly during sleep in the OSA group, likely due to compression caused by the enlargement of the lateral pharyngeal walls.

In conclusion, there is evidence in the Passos et al. ${ }^{(3)}$ study pointing to enlargement of the lateral pharyngea walls as the leading mechanism of obstruction in OSA, with a direct influence on the positioning of the soft palate and a likely accommodating movement of the upper tongue. On the basis of this alternative proposal, their study helps us understand, mechanistically, why positive airway pressure therapy should be initially applied through a nasal mask rather than an oronasal mask. ${ }^{(8,9)}$ It also helps explain the superiority of surgical techniques that reconstruct the lateral pharyngeal wall over traditional forms of uvulopalatopharyngoplasty. ${ }^{(10)}$ It seems that the key to future advancements in this field is to clarify why the lateral pharyngeal walls enlarge during sleep in individuals with OSA but not in healthy individuals.

\section{REFERENCES}

1. Guilleminault C, Tilkian A, Dement WC. The Sleep Apnea Syndromes, Annu Rev Med. 1976;27(1):465-84. https://doi.org/10.1146/annurev. me.27.020176.002341

2. De Vito A, Carrasco Llatas M, Ravesloot M, Kotecha B, De Vries N Hamans $E$, et al. European position paper on drug-induced sleep endoscopy: 2017 Update. Clin Otolaryngol. 2018;43(6):1541-1552. https://doi.org/10.1111/coa.13213

3. Passos UL, Genta PR, Marcondes BF, Lorenzi-Filho G, Gebrim EMMS. State-dependent changes in the upper airway assessed by multidetector CT in healthy individuals and during obstructive events in patients with sleep apnea. J Bras Pneumol. 2019;45(4):20180264 http://dx.doi.org/10.1590/1806-3713/e20180264

4. Gobbi R, Baiardi S, Mondini S, Cerritelli L, Piccin O, Scaramuzzino $\mathrm{G}$, et al. Technique and Preliminary Analysis of Drug-Induced Sleep Endoscopy With Online Polygraphic Cardiorespiratory Monitoring in Patients With Obstructive Sleep Apnea Syndrome. JAMA Otolaryngol Head Neck Surg. 2017;143(5):459-465. https://doi. org/10.1001/jamaoto.2016.3964

5. Olszewska E, Woodson BT. Palatal anatomy for sleep apnea surgery. Laryngoscope Investig Otolaryngol. 2019:4(1):181-187. https://doi. org/10.1002/lio2.238
6. Cho JH, Kim JK, Lee HY, Yoon JH. Surgical anatomy of human soft palate. Laryngoscope. 2013;123(11):2900-4. https://doi.org/10.1002/ lary. 24067

7. Glupker L, Kula K, Parks E, Babler W, Stewart K, Ghoneima A Three-dimensional computed tomography analysis of airway volume changes between open and closed jaw positions. Am J Orthod Dentofacial Orthop. 2015;147(4):426-34. https://doi.org/10.1016/j. ajodo.2014.11.025

8. Ebben MR, Milrad S, Dyke JP, Phillips CD, Krieger AC. Comparison of the upper airway dynamics of oronasal and nasal masks with positive airway pressure treatment using cine magnetic resonance imaging. Sleep Breath. 2016;20(1):79-85. https://doi.org/10.1007/ s11325-015-1187-x

9. Andrade RGS, Viana FM, Nascimento JA, Drager LF, Moffa A Brunoni AR, et al. Nasal vs Oronasal CPAP for OSA Treatment: a Meta-Analysis. Chest. 2018;153(3):665-674. https://doi.org/10.1016/j. chest.2017.10.044

10. Cahali MB, Formigoni GG, Gebrim EM, Miziara ID. Latera pharyngoplasty versus uvulopalatopharyngoplasty: a clinical polysomnographic and computed tomography measurement comparison. Sleep. 2004;27(5):942-50 